\title{
Distance Distributions of Short Polypeptides Recovered by Fluorescence Resonance Energy Transfer in the $10 \AA$ Domain
}

\author{
Harekrushna Sahoo, Danilo Roccatano, Martin Zacharias and Werner M. Nau* \\ School of Engineering and Science, International University Bremen, Campus Ring 1, \\ D-28759Bremen, Germany; w.nau@iu-bremen.de
}

\section{Contents}

1. Details on FRET Analysis

Page

1.1. Calculation of Förster Radii

S-2

1.2. Determination of FRET Efficiencies

S-2

1.3. Contribution of Diffusion-enhanced FRET

1.4. Determination of Effective Transfer Distances

1.5. Distribution Function Analysis

2. Materials and Methods

2.1. Materials

2.2. Spectroscopy

2.3. Molecular Dynamics (MD) Simulations

3. References 


\section{Details of FRET Analysis}

1.1. Calculation of Förster Radii. According to Förster theory, the rate of fluorescence resonance energy transfer $\left(k_{\mathrm{T}}\right)$ is related to the Förster radius $\left(R_{0}\right)$, excited-state lifetime of the donor in the absence of acceptor $\left(\tau_{\mathrm{D}}\right)$, and the donor-acceptor distance $(R)$ according to eq. 1 :

$$
k_{T}=\frac{1}{\tau_{D}}\left(\frac{R_{0}}{R}\right)^{6}
$$

$R_{0}$ is the distance at which one half of the singlet-excitation energy is transferred from the donor to the acceptor. $R_{0}$ is related to the orientation factor $\left(\kappa^{2}\right)$ between the electric transition dipoles of the donor and the acceptor as well as to their spectroscopic properties, namely the fluorescence quantum yield of the donor in the absence of acceptor $\left(\phi_{\mathrm{D}}\right)$ and the overlap integral $(J(\lambda))$. In addition, the refractive index $(n)$ of the chromophore environment enters the analytical expression for $R_{0}, c f$. eq. $2:^{1}$

$$
R_{0}=\left[8.79 \times 10^{-5}\left[\kappa^{2} n^{-4} \phi_{D} J(\lambda)\right]\right]^{1 / 6}
$$

The spectral overlap integral is calculated according to eq. 3, where $F_{\mathrm{D}}$ is the fluorescence intensity of the donor, $\varepsilon$ is the extinction coefficient of the acceptor with $\lambda$ as integration parameter. The unit of $J(\lambda)$ is typically given as $\mathrm{M}^{-1} \mathrm{~cm}^{-1} \mathrm{~nm}^{4}$.

$$
J(\lambda)=\frac{\int F_{D}(\lambda) \varepsilon(\lambda) \lambda^{4} d \lambda}{\int F_{D}(\lambda) d \lambda}
$$

In the present work, corrected fluorescence and UV absorption spectra were employed to calculate $J$ and the integration was performed between $290-530 \mathrm{~nm}$. In detail, fluorescence spectra were measured for the donor-only substituted peptide (Trp-(GlySer) $\left.{ }_{4}-\mathrm{OH}\right)$, and absorption spectra for the acceptor-only substituted peptide $\left((\text { GlySer})_{6}-\right.$ Dbo- $\left.\mathrm{NH}_{2}\right)$, both in the respective solvent (Figure 1 of main text). For the calculation of $R_{0}$, the fluorescence quantum yields of the Trp-labeled peptides were determined by relative measurements, using the amino acid $\operatorname{Trp}$ as reference $\left(\Phi_{\mathrm{f}}=0.13 \text { in water }\right)^{2}$ Table S-1 reveals that the $R_{0}$ values show slight variations between the free and peptide-bound chromophores, and depending on solvent, which is the result of slight variations in the photophysical properties. For example, when attached to a peptide, Dbo shows a small bathochromic shift (from 365 to $369 \mathrm{~nm}$ ) and increase in extinction coefficient (from 50 to $66 \mathrm{M}^{-1} \mathrm{~cm}^{-1}$ ); Trp shows a bathochromic shift (from 355 to $357 \mathrm{~nm}$ ) and a decrease in fluorescence quantum yield (from 0.13 to 0.07). The observed decrease in fluorescence quantum yield (and fluorescence lifetime, see below) is characteristic for the $N$-terminal attachment of Trp in peptides and has been related to variations in the population of different rotamers. ${ }^{3}$ 
The apparent refractive index relevant for FRET in large proteins is generally presumed to be higher than that in water. ${ }^{2,4}$ To experimentally assess the refractive index "felt" by the chromophores in the short peptides, we have representatively determined the oscillator strength and radiative decay rate of the Dbo chromophore in the (GlySer) $)_{4}-\mathrm{Dbo}-\mathrm{NH}_{2}$ peptide, because both photophysical parameters are known to be directly related to the refractive index (polarizability) of the environment. ${ }^{5,6}$ The resulting refractive index $\left(1.340 \pm 0.004\right.$, used in the $R_{0}$ calculations in water), determined from the previously established correlations, 5,6 was higher than that of water, but fell below the values frequently recommended to be used for FRET processes in large proteins (1.4-1.6). ${ }^{4,7}$ Corrections due to differences in refractive index are expected to become less relevant in protic organic solvents, such that we have directly used the solvent refractive indices for ethanol and propylene glycol.

Table S-1: Förster radii for different Trp/Dbo FRET pairs in different solvents

\begin{tabular}{llccc}
\hline \hline donor/acceptor & solvent & $J /\left(10^{11} \mathrm{M}^{-1} \mathrm{~cm}^{-1} \mathrm{~nm}^{4}\right)$ & $\Phi_{\mathrm{D}}$ & $R_{0} / \AA$ \\
\hline \multirow{2}{*}{ Trp/DBO } & $\mathrm{H}_{2} \mathrm{O}$ & 4.15 & 0.13 & 10.0 \\
& ethanol & 4.67 & 0.11 & 9.8 \\
& propylene glycol & 4.45 & 0.22 & 10.2 \\
\hline \multirow{2}{*}{ Trp-peptide $/$ DBO } & $\mathrm{H}_{2} \mathrm{O}$ & 4.16 & 0.07 & 8.9 \\
& ethanol & 4.12 & 0.07 & 8.8 \\
& propylene glycol & 4.09 & 0.16 & 9.5 \\
\hline \multirow{2}{*}{ Trp/peptide-Dbo ${ }^{\mathrm{b}}$} & $\mathrm{H}_{2} \mathrm{O}$ & 5.07 & 0.13 & 10.3 \\
& ethanol & 4.60 & 0.11 & 9.8 \\
& propylene glycol & 4.41 & 0.22 & 10.2 \\
\hline \multirow{2}{*}{ Trp-peptide $/$ peptide-Dbo $^{\mathrm{b}}$} & $\mathrm{H}_{2} \mathrm{O}$ & 5.09 & 0.07 & 9.2 \\
& ethanol & 3.96 & 0.07 & 8.8 \\
& propylene glycol & 4.01 & 0.16 & 9.5 \\
\hline \hline
\end{tabular}

${ }^{\mathrm{a}} \operatorname{Trp}-(\mathrm{GlySer})_{4}-\mathrm{OH} .^{\mathrm{b}}(\mathrm{GlySer})_{6}-\mathrm{Dbo}-\mathrm{NH}_{2}$.

The orientation factor $\left(\kappa^{2}\right)$ depends on the relative alignment of the transition dipoles of donor and acceptor to each other, which in the case of $\mathrm{DBO}$ is aligned along the $\mathrm{N}=\mathrm{N} \pi$ bond (parallel to the associated p orbitals) and for Trp diagonally across the indole ring system. ${ }^{8}$ The investigated peptides are structureless and flexible, ${ }^{9}$ such that randomized donor/acceptor orientations are expected in the 
ground state. This was confirmed by MD calculations in water (see below), which afforded completely randomized angles between the transition dipoles, i.e., the characteristic angular parameter $\left[\kappa_{M D}^{2}=\left(\sin \theta_{D} \sin \theta_{A} \cos \Phi_{D A}-2 \cos \theta_{D} \cos \theta_{A}\right)^{2}\right.$, where $\theta_{\mathrm{D}}$ and $\theta_{\mathrm{A}}$ are the angles of the donor and acceptor transition dipole moments to the chromophore distance vector $R$ and $\Phi_{\mathrm{DA}}$ is the dihedral angle between the two transition dipole moments] was found to be $0.66 \pm 0.02$ for three selected peptides ( $n$ $=0,1$, and 2), while the ideal value is 0.666. Accordingly, the orientation of the dipoles is expected to be randomized when excitation occurs. In such cases, the $\kappa^{2}$ value depends primarily on whether conformational changes within the donor excited-state lifetime occur and how fast. The limits of $\kappa^{2}$ can therefore be set between 0.666 (rapid dynamic averaging within the donor excited-state lifetime) and 0.476 (no conformational changes within the donor excited-state lifetime). ${ }^{10}$ For our calculations, we have set $\kappa^{2}$ as $2 / 3$ in all solvents. In propylene glycol as most viscous solvent, a somewhat smaller $\kappa^{2}$ value is most likely to apply, but even the choice of the lower limit of 0.476 would only lead to a small (6\% smaller) systematic error in the fitted distances (see below) as a consequence of a ca. $6 \%$ smaller $R_{0}$ value. In this context, it should also be mentioned that the choice of a different refractive index (see above) would not significantly affect the results either, due to the square-root-of-six dependence in eq. 2, which dampens the effect of small variations.

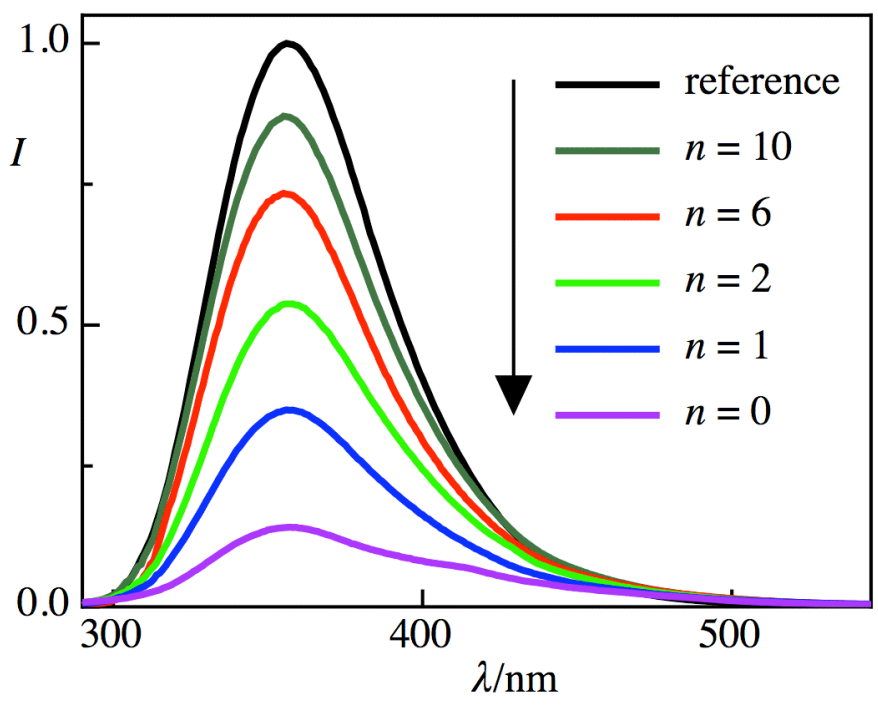

Figure S-1. Steady-state emission spectra of Trp-(GlySer $)_{n}-$ Dbo- $\mathrm{NH}_{2}$ peptides in $\mathrm{H}_{2} \mathrm{O}$ relative to the fluorescence decay of the Trp-(GlySer) ${ }_{4}-\mathrm{OH}$ reference peptide lacking the acceptor.

1.2. Determination of FRET Efficiencies. To assess the efficiency of energy transfer, the fluorescence intensity of the donor-acceptor-substituted peptide $\left(I_{\mathrm{DA}}\right)$ and the intensity of the donor-only-substituted 
peptide $\left(I_{\mathrm{D}}\right)$ as well as the associated fluorescence lifetimes ( $\tau_{\mathrm{DA}}$ and $\left.\tau_{\mathrm{D}}\right)$ were determined. It should be noted that the nanosecond time-resolved fluorescence decays were non-monoexponential in all cases, but they could be well fitted by biexponential decay functions. Non-monoexponential Trp fluorescence lifetimes are in fact a characteristics of this intrinsically fluorescent amino acid even in the absence of acceptors. ${ }^{11,12}$ The lifetimes were consequently determined as average lifetimes ${ }^{13,14}$ by taking into account the respective lifetime $\left(\tau_{\mathrm{i}}\right)$ and its relative contribution $\left(\phi_{\mathrm{i}}\right)$ in the form of a normalized preexponential factor $\left(\alpha_{\mathrm{i}}\right), c f$. eq . 4. Representative fluorescence spectra and time-resolved fluorescence decays are shown in Figures S-1 and S-2.

$$
\tau_{\text {avg }}=\sum \alpha_{i} \tau_{i} \text { with } \sum \alpha_{i} \equiv 1
$$
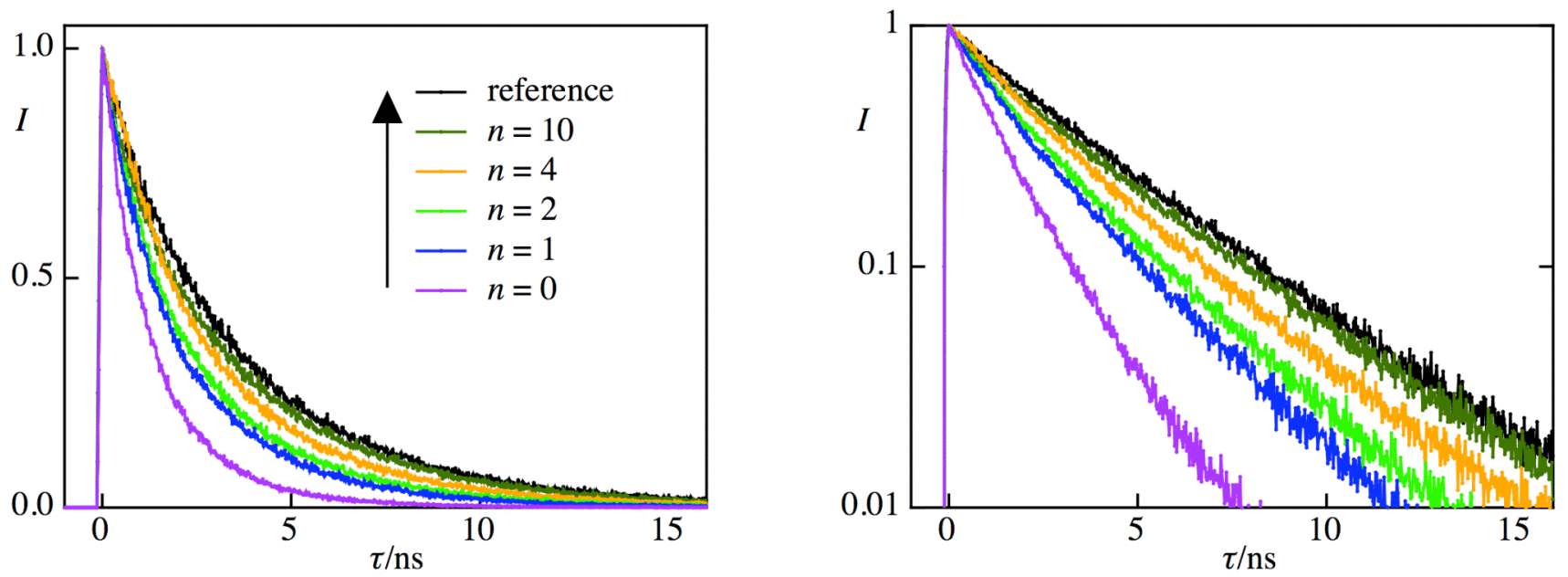

Figure S-2. Time-resolved fluorescence decays of Trp-(GlySer) ${ }_{\mathrm{n}}-\mathrm{Dbo}-\mathrm{NH}_{2}$ peptides in propylene glycol (normalized, linear intensity scale on the left and logarithmic one on the right) relative to the fluorescence decay of the Trp-(GlySer) $)_{4}-\mathrm{OH}$ reference peptide lacking the acceptor.

The pertinent FRET efficiencies derived from steady-state fluorescence measurements $\left(E_{\mathrm{ss}}\right)$ as well as those derived from time-resolved measurements $\left(E_{\mathrm{tr}}\right)$ were subsequently obtained from eqs. 5 and 6 . The difference (ca. $10 \%)$ was attributed to the fraction undergoing static quenching $\left(F=E_{\mathrm{ss}}-E_{\mathrm{tr}}\right.$, see main text) and assigned to the fraction of conformations which are in contact when excitation occurs. Close inspection shows, in fact, that a trend of decreasing static quenching with increasing peptide length applied, which is also expected from simple polymer models, ${ }^{15,16}$ i.e., longer peptides have a lower tendency to exist as a pre-formed loop structure. An exception was the shortest dipeptide $(n=0)$, which has a smaller fraction of static quenching than the next larger analogues $(n=1,2)$. This is presumably related to the higher intrinsic rigidity and steric hindrance effects in the shortest peptide, 
which is independently reflected in its longer persistence length (see main text) and its slower rate constant for end-to-end collision (loop formation). ${ }^{9}$

$$
\begin{aligned}
& E_{s S}=1-\left(I_{D A} / I_{D}\right) \\
& E_{t r}=1-\left(\tau_{D A} / \tau_{D}\right)
\end{aligned}
$$

It should be noted that the acceptor (Dbo) is fluorescent, but its fluorescence could not be employed to independently assess the FRET efficiency. First, the emission of Dbo in the peptide $\left(\lambda_{\max }\right.$ ca. $430 \mathrm{~nm}$ ) could not be spectrally resolved from the Trp fluorescence, and second, back quenching of the excited Dbo by the amino acid Trp applies, which itself is peptide-length dependent due to a collision-induced (exciplex) quenching mechanism. ${ }^{9}$ The latter is expected to greatly reduce the intensity of the Dbo emission, such that the fluorescence of the acceptor appeared merely as a small shoulder at ca. $420 \mathrm{~nm}$ (Figure S-2).

\subsection{Contribution of Diffusion-enhanced FRET. Diffusion-enhanced FRET describes the} enhancement of energy transfer due to mutual donor-acceptor diffusion within the excited-state lifetime of the probe. ${ }^{17,18}$ Diffusion can bring remote donor-acceptor pairs closer together during the excitedstate lifetime, such that the overall probability of FRET increases. The reverse process, namely diffusion apart to separate donor-acceptor pairs which are already in close proximity, is less relevant, because the excited-state lifetimes of these pairs are very short due to efficient FRET, which renders large spatial displacements during the excited-state lifetime less likely than for the remote pairs. The importance of diffusion-enhanced FRET can be semiempirically evaluated by calculating the term $D_{0} \tau_{0} / R_{\text {avg }}^{2}{ }^{17}$ which provides the ratio of the average displacement due to diffusion within the (unquenched) donor fluorescence lifetime $\left(D_{0} \tau_{0}\right)$ to the squared average distance $\left(R_{\text {avg }}^{2}\right)$ of the respective peptide. Depending on whether the term is much smaller or larger than 1, diffusionenhanced FRET can be neglected or becomes dominant, respectively. Taking the donor lifetime $\left(\tau_{0}\right)$ as $1.32 \mathrm{~ns}$ in water (the lifetime of the donor-only-labeled peptide Trp-(GlySer $\left.)_{4}-\mathrm{OH}\right)$ and the mutual diffusion coefficient of the chain ends $\left(D_{0}\right)$ as $5.4 \times 10^{-7} \mathrm{~cm}^{2} \mathrm{~s}^{-1}$, the value previously calculated for the Trp-(GlySer) $)_{4}$-Dbo- $\mathrm{NH}_{2}$ peptide in $\mathrm{H}_{2} \mathrm{O},{ }^{19}$ and $R_{\text {avg }}$ as ca. $10 \AA$ (see Table 1 in main text) one obtains a value of ca. 0.07, which suggests that diffusion-enhanced FRET is not dominant, but cannot be neglected in $\mathrm{H}_{2} \mathrm{O}$ as solvent. A slightly larger value (ca. 0.13) results for ethanol due to its comparable solvent viscosity but longer Trp fluorescence lifetime (2.35 ns). In propylene glycol, on the other hand, a value $<0.005$ is expected due to its 45 times lower viscosity (ca. 45 times smaller $D_{0}$ value), such that diffusion-enhanced FRET can be neglected in this solvent. In general, mutual diffusion is considered to be insignificant for peptides in propylene glycol, unless the donor lifetimes are more than one order of 
magnitude longer (ca. $50 \mathrm{~ns}$ ), or unless elevated temperatures are being employed. ${ }^{18}$ In summary, diffusion of the donor-acceptor pairs within the short excited-state lifetime of Trp should be insignificant in propylene glycol, but become sizable in non-viscous solvents like water and ethanol.

1.4. Determination of Effective Transfer Distances. In systems with a fixed and uniform donoracceptor distance $(R)$, e.g., in site-specifically donor-acceptor labeled structured proteins, $R$ can be directly calculated from the energy transfer efficiency using eq. 7.

$$
R^{6}=\frac{R_{0}^{6}(1-E)}{E}
$$

In the case of structureless polymers, including the random-coiled flexible peptides examined herein, eq. 7 does not strictly apply. Instead, the distribution function of the biopolymer ${ }^{2}$ (and in limiting cases also diffusion-enhanced FRET) needs then to be considered. However, also in these cases eq. 7 allows the calculation of "effective" transfer radii $\left(R_{\text {eff }}\right)$ from the steady-state FRET data, which are listed in Table S-2. On one hand, the $R_{\text {eff }}$ values reveal useful trends, e.g., they increase systematically with increasing peptide length. Most importantly, the $R_{\text {eff }}$ values in water and ethanol are significantly smaller than those in propylene glycol. This reveals the sizable contribution of diffusionenhanced FRET in the two nonviscous solvents (see above), which increases the energy transfer efficiency and, therefore, leads to smaller $R_{\text {eff }}$ values. Detailed inspection also shows that the values in ethanol are consistently smaller than the corresponding ones in water (Table S-2). This can be nicely rationalized in terms of the enhanced (factor 2) contribution of diffusion-enhanced FRET in this solvent, which is a direct consequence of the longer Trp fluorescence lifetime in ethanol (see above).

The FRET efficiencies derived from the time-resolved data could not be directly used to calculate $R_{\text {eff }}$ values because they do not include the fraction of conformations in contact (static quenching). The experimental fraction of static quenching can be considered according to $R_{\text {eff }}=(1-F) R_{\mathrm{tr}}+F R_{\mathrm{vdW}}$, where $F$ is the fraction of conformations in contact and $R_{\mathrm{vdW}}$ is the contact distance. The latter can be taken from molecular dynamics calculations, which suggest that a fraction of peptide conformations is in a van-der-Waals contact distance of ca. $4.0 \AA^{20,21}$ The $R_{\text {eff }}$ values from the time-resolved measurements "corrected" in this manner are in excellent agreement with the $R_{\text {eff }}$ values from the steady-state experiments (Table S-2). The two complementary experiments do therefore provide mutually consistent effective transfer distances, which supports the interpretation of the variations in the transfer efficiencies in terms of static quenching. 
Table S-2. Effective transfer distances in different solvents

\begin{tabular}{|c|c|c|c|c|c|c|c|c|c|c|c|c|c|}
\hline \multirow{2}{*}{$n$} & \multirow{2}{*}{ method } & \multicolumn{4}{|c|}{ water } & \multicolumn{4}{|c|}{ ethanol } & \multicolumn{4}{|c|}{ propylene glycol } \\
\hline & & $\tau_{\mathrm{DA}} / \mathrm{ns}^{\mathrm{a}}$ & $E$ & $R_{\mathrm{tr}} / \AA$ & $R_{\mathrm{eff}} / \AA$ & $\tau_{\mathrm{DA}} / \mathrm{ns}^{\mathrm{b}}$ & $E$ & $R_{\mathrm{tr}} / \AA$ & $R_{\mathrm{eff}} / \AA$ & $\tau_{\mathrm{DA}} / \mathrm{ns}^{\mathrm{c}}$ & $E$ & $R_{\mathrm{tr}} / \AA$ & $R_{\text {eff }} / \AA$ \\
\hline \multirow[t]{2}{*}{0} & steady-state & & 0.84 & & 7.0 & & 0.80 & & 7.0 & & 0.72 & & 8.1 \\
\hline & time-resolved & 0.41 & 0.69 & 8.1 & 7.5 & 0.64 & 0.73 & 7.4 & 7.2 & 1.28 & 0.60 & 8.9 & 8.3 \\
\hline \multirow[t]{2}{*}{1} & steady-state & & 0.65 & & 8.3 & & 0.64 & & 8.0 & & 0.56 & & 9.1 \\
\hline & time-resolved & 0.58 & 0.56 & 8.9 & 8.5 & 0.93 & 0.61 & 8.2 & 8.0 & 1.89 & 0.40 & 10.2 & 9.2 \\
\hline \multirow[t]{2}{*}{2} & steady-state & & 0.46 & & 9.5 & & 0.51 & & 8.7 & & 0.45 & & 9.9 \\
\hline & time-resolved & 0.76 & 0.42 & 9.7 & 9.5 & 1.22 & 0.48 & 8.9 & 8.8 & 2.14 & 0.32 & 10.8 & 9.9 \\
\hline \multirow[t]{2}{*}{4} & steady-state & & 0.39 & & 9.9 & & 0.36 & & 9.7 & & 0.32 & & 10.8 \\
\hline & time-resolved & 0.95 & 0.28 & 10.9 & 10.1 & 1.55 & 0.34 & 9.8 & 9.7 & 2.51 & 0.21 & 11.9 & 11.0 \\
\hline \multirow[t]{2}{*}{6} & steady-state & & 0.27 & & 10.9 & & 0.26 & & 10.5 & & 0.24 & & 11.5 \\
\hline & time-resolved & 1.03 & 0.22 & 11.4 & 11.1 & 1.85 & 0.22 & 10.9 & 10.6 & 2.61 & 0.18 & 12.3 & 11.8 \\
\hline \multirow[t]{2}{*}{10} & steady-state & & 0.13 & & 12.7 & & $d$ & & & & 0.12 & & 13.3 \\
\hline & time-resolved & 1.18 & 0.10 & 13.3 & 13.0 & & & & & 2.92 & 0.08 & 14.4 & 14.0 \\
\hline
\end{tabular}

${ }^{a}$ Average lifetime of reference peptide without acceptor was $\tau_{\mathrm{D}}=1.32 \mathrm{~ns}$. ${ }^{\mathrm{b}}$ Average lifetime of reference peptide without acceptor was $\tau_{\mathrm{D}}=2.35 \mathrm{~ns} .{ }^{\mathrm{c}}$ Average lifetime of reference peptide without acceptor was $\tau_{\mathrm{D}}=3.17 \mathrm{~ns}$.

${ }^{\mathrm{d}}$ Peptide insufficiently soluble in ethanol. 
1.5. Distribution Function Analysis. Distance distributions were recovered from the time-resolved donor fluorescence decays in propylene glycol in the absence of diffusion-enhanced FRET. ${ }^{22,23}$ Assuming that deactivation of the acceptors occurs only by FRET and the natural decay pathways, eq. 8 applies, where $P(R)$ presents the distance distribution function of the biopolymer and $\alpha_{\mathrm{i}}$ and $\tau_{\mathrm{i}}$ are the preexponential factors and lifetimes of the decay components in the absence of an acceptor.

$$
I_{D A}(t)=I_{0} \int P(R) \sum_{i} \alpha_{i} \exp \left[-\frac{t}{\tau_{D, i}}\left(1+\left(\frac{R_{0}}{R}\right)^{6}\right)\right] d R
$$

We employed a probability distribution based on the wormlike chain model in eq. $9,{ }^{24}$ which was implemented within a numerical integration procedure for fitting. ${ }^{25}$ Wormlike distributions fulfil implicitly two important boundary conditions, which are not necessarily met by alternative distribution functions, e.g., a (symmetrical) Gaussian function (see below). First, wormlike distributions do not extend to distances larger than that of the most extended conformation, and $b$ ) the distributions become negligibly small at distances below the van-der-Waals limit of ca. $4 \AA$. In eq. $9, N$ presents a normalization factor, $l_{\mathrm{p}}$ represents the persistence length, i.e., a measure of the rigidity of the peptide which characterizes essentially the half-width of the related distribution, and $l_{\mathrm{c}}$ is the contour length, which is related to the length of the peptide. The fittings were excellent and the recovered data are entered in Table 1 of the main text.

$$
P(R)=\frac{4 \pi N R^{2}}{l_{c}^{2}\left[1-\left(R / l_{c}\right)^{2}\right]^{9 / 2}} \exp \left(-\frac{3 l_{c}}{4 l_{p}\left[1-\left(R / l_{c}\right)^{2}\right]}\right)
$$

We also performed the fitting using a Gaussian distribution function, ${ }^{2}$ which is characterized by a mean distance $\left(R_{\text {mean }}\right)$ and a standard deviation $(\sigma), c f$. eq 10 . The latter presents a measure of the broadness of the distribution and is related to its half-width (full width at half-maximum, $h w$ ) as $h w=$ $2.354 \sigma$. The fittings according to the Gaussian distribution were of comparable quality as those for the wormlike chain model (Table S-3). The obtained distributions were also reasonable, e.g., they did not significantly extend to distances below van-der-Waals contact. For comparison, a previous study on a hexaglycine peptide performed with a longer-range FRET pair $\left(R_{0} \approx 25 \AA\right)$ has afforded a Gaussian distribution $\left(R_{\text {mean }}=10.7 \AA\right.$ and $\left.h w=18.1 \AA\right)$ which extended to distances far below $4 \AA^{23}$

$$
P(R)=\frac{1}{\sigma \sqrt{2 \pi}} \exp \left[-\frac{1}{2}\left(\frac{R-R_{\text {mean }}}{\sigma}\right)\right]
$$

The average distances $\left(R_{\text {avg }}\right)$ obtained from a combination of the Gaussian distributions and the short-distance contributions due to static quenching (Figure S-3) were found to be larger (Table S-3) 
than those obtained for the wormlike chain model (Table 1 in main text), with the exception of the shortest dipeptide.

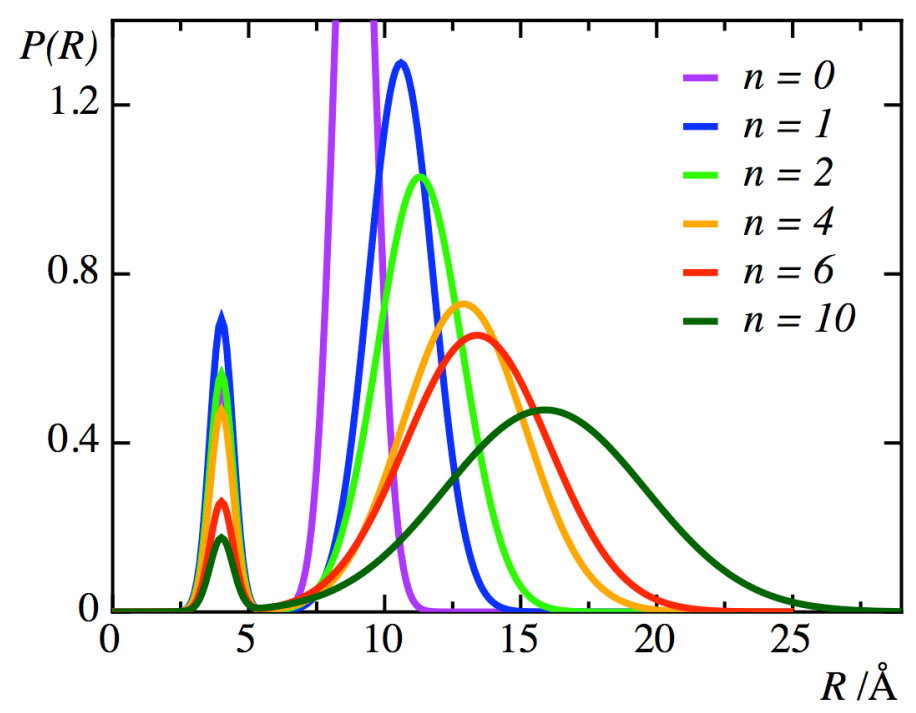

Figure S-3. Distribution functions recovered from the analysis of the time-resolved fluorescence decays in propylene glycol according to a Gaussian distribution-dependent FRET kinetics; function for $n=0$ is cut off for clarity; the short-distance peak represents the static quenching component and was placed at $4 \AA$ (1.0 A half-width).

Table S-3. Half-widths $(h w)$ and mean distances $\left(R_{\text {mean }}\right)$ characterizing a Gaussian-type distribution function obtained from fitting of the time-resolved fluorescence decays in propylene glycol according to eq. 10 and average distances $\left(R_{\text {avg }}\right)$ including contact conformations.

\begin{tabular}{llll}
\hline \hline$n$ & $h w /(\AA)$ & $R_{\text {mean }} /(\AA)$ & $R_{\text {avg }} /(\AA)$ \\
\hline 0 & 1.70 & 8.9 & 8.3 \\
1 & 2.80 & 10.6 & 9.6 \\
2 & 3.66 & 11.3 & 10.4 \\
4 & 5.29 & 12.9 & 12.0 \\
6 & 6.22 & 13.4 & 12.8 \\
10 & 8.70 & 15.9 & 15.4 \\
\hline \hline
\end{tabular}

It should be noted that no satisfactory fittings could be achieved for water and ethanol by either distribution function. In addition, in the case of the Gaussian distribution, the recovered half-width 
were far too small (0.01-0.7 $\AA$ ). This phenomenon, namely that the fitting according to a Gaussian distribution function gives apparently too small distributions (as would be expected for fixed donor/acceptor distances) whenever diffusion-enhanced FRET interferes, has been recognized previously. ${ }^{18}$ The failure to fit the time-resolved fluorescence decays in the two non-viscous solvents by simple distribution function models presents another manifestation of the interference of diffusionenhanced FRET (see above) in these solvents.

\section{Materials and Methods}

2.1. Materials. Details on the synthesis of the probe (Fmoc-protected Dbo) and its suitability in solidphase peptide synthesis have been previously reported. ${ }^{9}$ The donor/acceptor containing peptides, Trp$(\text { GlySer })_{n}-$ Dbo- $\mathrm{NH}_{2}(n=0-10),{ }^{9}$ as well as the reference peptide without acceptor, Trp-(GlySer $)_{4}-\mathrm{OH}$, were commercially synthesized (Biosyntan, Berlin) in $>96 \%$ purity. Nanopure water (PURELAB) was used. Propylene glycol (Fluka) and ethanol (Riedel-de Haën) were spectroscopic grade and used as received.

2.2. Spectroscopy. All measurements were performed at ambient temperature $\left(25{ }^{\circ} \mathrm{C}\right)$. The fluorescence lifetimes of the GlySer-peptides were measured in $\mathrm{H}_{2} \mathrm{O}$, ethanol and propylene glycol under air by time-correlated single-photon counting (FLS920, Edinburgh Instruments Ltd.) using a PicoQuant pulsed LED (PLS-280, $\lambda_{\mathrm{exc}}=280 \mathrm{~nm}, \lambda_{\mathrm{obs}}=350 \mathrm{~nm}$, fwhm ca. $450 \mathrm{ps}$ ) for excitation. Corrected steady-state emission spectra and intensities $\left(\lambda_{\text {exc }}=280 \mathrm{~nm}\right)$ were recorded with a spectrofluorometer (Cary Eclipse, Varian). The optical density of the peptides at the excitation wavelength was adjusted to ca. 0.10 for the steady-state fluorescence measurements by using a UVvisible spectrophotometer (Cary 4000, Varian); optically matched ( $\pm 5 \%$ ) solutions containing a reference peptide with known quantum yield were employed to calculate the relative fluorescence quantum yields, and residual variations in optical density as well as variations in solvent refractive index were corrected for as described previously. ${ }^{6}$

2.3. Molecular Dynamics (MD) Simulations. The starting structures of the $n=0-6$ peptides were modeled in an extended conformation with a protonated amino group, which is expected to dominate in neutral aqueous solution. ${ }^{9}$ Details on the parameterization of the Dbo residue will be described elsewhere. The peptides were solvated with water molecules in sufficiently periodic cubic boxes to contain the molecule and $0.9 \mathrm{~nm}$ of solvent on all sides. All solvent molecules within $0.15 \mathrm{~nm}$ of any amino acid atom were removed. Since the resulting protonation state of the peptide gives rise to a total charge of +1 , a single chloride counterion was added by replacing a water molecule in the region of 
most negative electrical potential to provide a neutral simulation box. All the systems were subsequently energy minimized with a steepest descent method for 1000 steps. The temperature was maintained close to the intended values by weak coupling to an external temperature bath ${ }^{26}$ with a coupling constant of $0.1 \mathrm{ps}$. The peptide and the rest of the system were coupled separately to the temperature bath. The pressure was kept constant by weak coupling to a bath of constant pressure $\left(p_{0}=\right.$ 1 bar, coupling time $\tau_{\mathrm{p}}=0.5 \mathrm{ps}$ ). ${ }^{26}$ The simple point charge (SPC) ${ }^{27}$ model, LINCS algorithm (to constrain all bond lengths), ${ }^{28}$ and SETTLE algorithm (for water molecules) ${ }^{29}$ were used. A dielectric permittivity, $\varepsilon=1$, and a time step of 2 fs were used. A twin range cut-off was used for the calculation of the non-bonded interactions. The short-range cut-off radius was set to $0.8 \mathrm{~nm}$ and the long-range cut-off radius to $1.4 \mathrm{~nm}$ for both Coulomb and Lennard-Jones interactions. The cut-off values are the same as those used for the GROMOS96 force field parameterization. ${ }^{30}$ No reaction field corrections beyond the long-range cut-off were included in the cut-off simulations. Interactions within the shortrange cut-off were updated every time step whereas interactions within the long-range cut-off were

updated every 5 time steps together with the pair-list. As was shown in a previous paper, ${ }^{21}$ the use of the Particle Mesh Ewalds method was expected to produce only marginal improvements, which did not justify the associated increase of computational time required for the simulation.

All atoms were given an initial velocity obtained from a Maxwellian distribution at the desired initial temperature. All simulations were equilibrated by 50-ps MD runs with position restraints on the peptide to allow the relaxation of the solvent molecules. For the shortest peptides with $n=0-3$, after equilibration, two $150 \mathrm{~ns}$ production run simulations were performed. For the longer peptides $(n=4$ and 6) only one $100 \mathrm{~ns}$ long simulation was performed. All MD runs and the analysis of the trajectories were performed using the GROMACS software package. ${ }^{31,32}$

\section{References}

1. Selvin, P. R., Methods in Enzymology: Biochemical Spectroscopy 1995, 246, 300-334.

2. Lakowicz, J. R., Principles of Fluorscence Spectroscopy. Kluwer Academic/Plenum: New York, 1999.

3. Chen, R. F.; Knutson, J. R.; Ziffer, H.; Porter, D., Biochemistry 1991, 30, 5184-5195.

4. Toptygin, D.; Savtchenko, R. S.; Meadow, N. D.; Roseman, S.; Brand, L., J. Phys.Chem. B 2002, 106, 3724-3734.

5. Marquez, C.; Nau, W. M., Angew. Chem. Int. Ed. 2001, 40, 4387-4390.

6. Mohanty, J.; Nau, W. M., Photochem. Photobiol. Sci. 2004, 3, 1026-1031. 
7. Lakowicz, J. R., Principles of Fluorscence Spectroscopy. Kluwer Academic/Plenum: New York, 1999; p p. 368-369.

8. $\quad$ Eftink, M. R.; Selvidge, L. A.; Callis, P. R.; Rehms, A. A., J. Phys. Chem. 1990, 94, 3469-3479.

9. Hudgins, R. R.; Huang, F.; Gramlich, G.; Nau, W. M., J. Am. Chem. Soc. 2002, 124, 556-564.

10. Klostermeier, D.; Millar, D. P., Biopolymers 2002, 61, 159-179.

11. Creed, D., Photochem. Photobiol. 1984, 39, 537-562.

12. Blancafort, L.; Gonzalez, D.; Olivucci, M.; Robb, M. A., J. Am. Chem. Soc. 2002, 124, 6398-6406.

13. Wu, P.; Brand, L., Biochemistry 1992, 31, 7939-7947.

14. Millar, D. P., Methods in Enzymology, Part C: Energetics of Biological Macromolecules 2000, $323,442-459$.

15. Flory, P. J.; Semlyen, J. A., J. Am. Chem. Soc. 1966, 88, 3209-3212.

16. Nau, W. M.; Huang, F.; Wang, X. J.; Bakirci, H.; Gramlich, G.; Marquez, C., Chimia 2003, 57, 161-167.

17. Stryer, L.; Thomas, D. D.; Meares, C. F., Ann. Rev. Biophys. Bioeng. 1982, 11, 203-222.

18. Lakowicz, J. R., Principles of Fluorscence Spectroscopy. Kluwer Academic/Plenum: New York, 1999; p 411-416.

19. Wang, X.; Bodunov, E. N.; Nau, W. M., Opt. Spectrosc. 2003, 25, 560-570.

20. Yeh, I.-C.; Hummer, G., J. Am. Chem. Soc. 2002, 124, 6563-6568.

21. Roccatano, D.; Nau, W. M.; Zacharias, M., J. Phys. Chem. B 2004, 108, 18734-18742.

22. Cantor, C. R.; Pechukas, P., Proc. Natl. Acad. Sci. USA 1971, 68, 2099-2101.

23. Lakowicz, J. R.; Wiczk, W.; Gryczynski, I.; Johnson, M. L., Proc. SPIE 1990, 1204, 192-205.

24. Thirumalai, D.; Ha, B.-Y. In Theoretical and Mathematical Models in Polymer Research, Grossberg, A., Ed. Academia: New York, 1998; pp 1-35.

25. ProFit 6.0.3 ed., 2005; QuantumSoft: Zürich, Switzerland.

26. Berendsen, H. J. C.; Postma, J. P. M.; van Gunsteren, W. F.; DiNola, A.; Haak, J. R., J. Chem. Phys. 1984, 81, 3684-3690.

27. Berendsen, H. J. C.; Grigera, J. R.; Straatsma, T. P., J. Phys. Chem. 1987, 91, 6269-6271.

28. Hess, B.; Bekker, H.; Berendsen, H. J. C.; Fraaije, J. G. E. M., J. Comp. Chem. 1997, 18, $1463-$ 1472.

29. Miyamoto, S.; Kollman, P. A., J. Comp. Chem. 1992, 13, 952-962.

30. van Gunsteren, W. F.; Billeter, S. R.; Eising, A. A.; Hünenberger, P. H.; Krüger, P.; Mark, A. E.; Scott, W. R. P.; Tironi, I. G., Biomolecular Simulation: The GROMOS96 Manual and User Guide. vdf Hochschulverlang: ETH Zürich, Switzerland, 1996.

31. Berendsen, H. J. C.; van der Spoel, D.; van Drunen, R., Comp. Phys. Comm. 1995, 91, 43-56.

32. Lindahl, E.; Hess, B.; van der Spoel, D., J. Mol. Mod. 2001, 7, 306-317. 